\title{
Acerca del documento SOCHICAR sobre la Adherencia farmacológica en la prevencion cardiovascular en Chile: ¿Qué más se necesita fuera del uso de la polipíldora?
}

\author{
Tomás Romero \\ University of California, San Diego \\ Institutional Review Board, Sharp Health Care, \\ San Diego, California
}

Señor Editor,

El reciente documento publicado en la Rev Chil de Cardiología por los miembros del Departamento de Prevención Cardiovascular de la Soc. Chilena de Cardiología aborda el complejo e importante tema de la Adherencia farmacológica en la prevención secundaria cardiovascular y discute publicaciones internacionales que, según ellos estiman, apoyan el uso en Chile de la polipíldora en la prevención secundaria cardiovascular ${ }^{1}$.

Sin embargo, en este detallado y extenso recuento de los diferentes factores involucrados en la adherencia farmacológica no se mencionan aspectos que han dificultado su evaluación hasta la fecha y que han sido analizados en múltiples publicaciones y también en un artículo recientemente publicado en la Rev Chil de Cardiología, entre los cuales se mencionan la falta de uniformidad en las metodologías utilizadas para medirla y la frecuente disparidad en las características de las poblaciones en que ha sido evaluada ${ }^{2,3}$. Además, es necesario destacar la importancia de considerar el tiempo durante el cual los pacientes están sometidos al tratamiento farmacológico, ya que es por todos conocida su influencia negativa en la adherencia, suficientemente res- paldada por la literatura pertinente ${ }^{4}$. Todas las medidas que se vayan adoptando, incluyendo la polipíldora, con la intención de mejorar la adherencia farmacológica deberán anticipar esta potencial influencia desfavorable que será cada vez mayor dado el esperable aumento de la prevalencia de enfermedades crónicas, y entre ellas la hipertension arterial y las enfermedades arterioscleróticas cardiovasculares, que traerá el envejecimiento progresivo de la población.

Como ilustración de la influencia del tiempo de tratamiento sobre la adherencia farmacológica me referiré a dos estudios nacionales recientes realizados independientemente en pacientes hipertensos seguidos en centros de atención de salud familiar (CESFAM) metropolitanos dependientes del Ministerio de Salud, que en su mayoría sirven a una población de nivel socio-económico predominantemente bajo. Uno de ellos reportó una adherencia farmacológica de $54 \%$ en hipertensos, seguidos por un período de 4 meses, mientras que el otro comprobó sólo $36.6 \%$ de adherencia en una población seguida por 12 meses; la adherencia se midió en ambos grupos utilizando el mismo método (cuestionario Morisky-Green-Levine version 4) ${ }^{5,6}$. En estos estudios la adherencia farmacológica estuvo, además, lejos de las cifras consideradas como "ideales" ( $80 \%$ o mayor) a pesar 
de que los pacientes estaban acogidos al Programa de Salud Cardiovascular del Ministerio de Salud, que implica acceso gratuito a los fármacos antihipertensivos, y a un seguimiento programado de controles periódicos libre de costos adicionales. Mejorar el acceso a la atención médica y a los fármacos requeridos es sin duda una meta indispensable en todo programa de salud pero estas experiencias destacan indirectamente la influencia pertinaz y no desentrañada hasta el día de hoy de los mecanismos a través de los cuales los factores socio-económicos se asocian a conductas y desenlaces en el terreno de la salud. ${ }^{7}$

Los autores del documento Sochicar describen en gran detalle los potenciales beneficios de mejorar la adherencia farmacológica en Chile mediante el uso de la polipíldora en la prevención cardiovascular secundaria pero también destacan los posibles escollos en lograrlo, inherentes a las esperables diferencias en la tolerancia individual de una píldora que incluye varios fármacos. En este escenario, y como lo sugieren las experiencias nacionales aludidas, futuros esfuerzos en aumentar la adherencia podrían también verse limitados si no van acompañados por un progreso en las características socio-económicas de la población chilena.

En síntesis, la opinión experta publicada hasta la fecha apoya el uso de la polipíldora para optimizar la adherencia farmacológica. Sin embargo son muchos los factores aun no resueltos en la prevención cardiovascular secundaria mas allá de la adherencia a los fármacos que incluyen entre otros, la inducción exitosa de cambios en los estilos de vida y hábitos perjudiciales después de un evento cardiovascular. Es conocida por todos la eficacia en lograrlo mediante programas de rehabilitación cardíaca, pero también su limitado uso actual a pesar de su demostrada utilidad. Estos aspectos han sido analizados en una publicación reciente de la Rev Chil. de Cardiol., destacando barreras y factores facilitadores en la adopción de estilos de vida favorables después de un infarto agudo del miocardio, existentes tanto entre los pacientes como en los proveedores de salud ${ }^{8}$.

Es claro que aún no contamos con la bala de plata para detener el avance de la enfermedad arteriosclerótica cardiovascular. ¿Será la polipíldora la respuesta?.

\section{Referencias}

1. Adherencia farmacológica y prevención secundaria cardiovascular: una de las principales barreras en el tratamiento de la enfermedad arteriosclerótica. Posición del Departamento de Pevención Cardiovascular de SOCHICAR. en el uso de la polipíldora en prevención secundaria. Rev.Chil. Cardiol. 2016;35:270-

2. OSTERBERG L, BLASCHKE T. Adherence to Medication. N Engl J Med 2005; 353: 487-97.

3. ROMERO T. Adherencia al tratamiento antihipertensivo: ¿por qué aún no despega, y en qué medida influye en el control de la Presión Arterial? Rev Chil. Cardiol 2014; 33: 210-214

4. VRIJENS B, VINCZE G, KRISTANTO P, URQUHART J, BURNIER M. Adherence to prescribed antihypertensive drug treatments: longitudinal study of electronically compiled dosing histories. BMJ. 2008; 336: 1114
5. VARLETA P, AKEL C, ACEVEDO M, SALINAS C, PINTO J, OPAZO V, et al. Prevalencia y determinantes de adherencia a terapia antihipertensiva en pacientes de la Región Metropolitana. Rev Med Chile 2015; 143: 569-576.

6. SANDOVAL D, CHACÓN J, MUÑOZ R, KOCH E, ROMERO T. Influence of psychosocial factors on adherence to antihypertensive drug therapy. Results from a Cardiovascular Health Program cohort followed in the Metropolitan Region of Santiago, Chile. Rev Med Chile 2014; 142: 1245-1252

7. MARMOT M. Social Determinants of Health Inequalities. Lancet.2005; 365: 1099-104.

8. BAMBS CE, SGOMBICH MX, LEIVA LE, BARAONA FE, MARGOZZINI PA, PIZARRO CA, et al. Facilitators and barriers to the adoption of healthy lifestyles after first myocardial infarction in Chile: A qualitative study. Rev Chil Cardiol 2015; 34: 163-174. 